A Simple Probe for SuperResolution Imaging of the Endoplasmic Reticulum in Living Cells

\author{
Journal Article \\ Author(s): \\ Rivera-Fuentes, Pablo; Halabi, Elias A.; Püntener, Salome \\ Publication date: \\ 2018-11 \\ Permanent link: \\ https://doi.org/10.3929/ethz-b-000307886
}

Rights / license:

In Copyright - Non-Commercial Use Permitted

Originally published in:

Helvetica Chimica Acta 101(11), https://doi.org/10.1002/hlca.201800165 


\title{
A Simple Probe for Super-Resolution Imaging of the Endoplasmic Reticulum in Living Cells
}

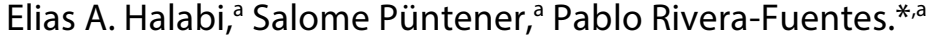 \\ aLaboratory of Organic Chemistry, ETH Zurich, Zurich, Switzerland. * Correspondence should be addressed to P. R.-F. (pablo.rivera- \\ fuentes@org.chem.ethz.ch).
}

Dedicated to François Diederich on the occasion of his retirement

\begin{abstract}
Super-resolution imaging of living cells can reveal nanoscopic details of live biological systems. The development of small-molecule fluorophores that allow optimal imaging conditions is key to enable live-specimen imaging with minimal invasiveness. In this study, we report a simple and non-toxic rhodamine-based diazoindanone probe compatible with direct stochastic optical reconstruction microscopy (d-STORM). Co-localization studies performed in human cervical cancer (HeLa) cells indicated that this probe targets the endoplasmic reticulum (ER). Photophysical experiments carried out in polyvinyl alcohol films revealed that each molecule yields a high number of photons before photodecomposition ( $80^{\prime} 000$ photons), allowing good localization precision $(42 \pm 12 \mathrm{~nm}$ ) in single-molecule localization experiments. Super-resolution imaging employing this photoactivatable probe permitted the visualization of nanoscopic pores within the network of tubules and sheets of the endoplasmic reticulum. We further analyzed this structure in three dimensions to distinguish pores from concave surfaces and built 3D reconstructions of these nanometric tubules and cisternae.
\end{abstract}

Keywords: super-resolution microscopy • endoplasmic reticulum • single-molecule localization microscopy • fluorophores

\section{Introduction}

Single molecule localization microscopy (SMLM) identifies sparsely distributed signals emitted from single fluorophores to reconstruct images with nanometric resolution. These light microscopy techniques enabled the observation of biological structures smaller than the diffraction limit of light $(\sim 250 \mathrm{~nm})$. $^{1}$ Because SMLM utilizes visible light, the greatest advantage over other highly resolved techniques such as electron microscopy is the possibility to image nanometric structures in live specimens and real time. ${ }^{2}$ Time-lapse reconstructions of superresolved single images can provide highly detailed movies of nanoscale interactions in real time, ${ }^{3}$ allowing for the observation of these processes with excellent spatial resolution.

The quality of live-cell super-resolution imaging depends on the properties of the fluorescent markers. Ideally, these dyes should diffuse into the cell and label structures with specificity. These probes must also be (photo)chemically stable and non-toxic. ${ }^{4}$ Additionally, the fluorophore must emit a large number of photons prior to photodecomposition because the resolution of the final image depends on the intensity of the signal of each individual molecule., ${ }^{5,6}$ The advantage of using smallmolecule fluorophores instead of fluorescent proteins is the easy tuning of the fluorophore, without the need for time intensive genetic manipulation.?

Among the many dyes that have been explored for super-resolution techniques, photoactivatable and photoswitchable xanthene dyes are privileged structures with excellent performance in single molecule detection. ${ }^{8,9}$ Xanthene dyes become photoactivatable by transforming them into spirocyclic diazoindanone derivatives. ${ }^{10}$ These derivatives respond to light of $405 \mathrm{~nm}$ to produce bright emissive dyes that are suitable for SMLM. We reported the use of diazoindanone rhodols as effective dual photoactivatable dyes that emit a fluorescent signal selectively in the presence of an active carboxylesterase. ${ }^{11}$ This method enables the detection of clusters of active enzymes because the photoactivated dyes form a covalent bond with their surroundings, which limits their diffusion and facilitates SMLM.

In this study, we report a photoactivatable rhodamine diazoindanone probe ER-1 that is membrane permeable and selectively targets fine structures of the endoplasmic reticulum (ER) at the periphery of the cell. Because the fluorescent product of ER-1 covalently attaches to surrounding proteins upon activation, it does not require any additional targeting strategies, enabling SMLM studies on mammalian living cells to observe dynamics of the ER at nanometric resolution. Thus, we provide a simple way to study the morphology of the ER and shine light on the time-dependent, three-dimensional rearrangement of these nanoscopic features. ${ }^{12}$

\section{Results and Discussion}

Target compound ER-1 was obtained by a three-step synthetic route starting from the condensation of trimellitic anhydride ethylester and 8hydroxyjulolidine in toluene to afford rhodamine $\mathbf{2}$ as a mixture of isomers 5 and 6 (Figure $1, a$ and Supporting Information). The diazoindanone rhodamine $\mathbf{3}$ was prepared by treatment of $\mathbf{2}$ with oxalyl chloride, followed by reaction with (trimethylsilyl)diazomethane under basic conditions. Saponification of $\mathbf{3}$ gave the corresponding ER-1 probe in good yields (Figure 1,a). In the absence of light $(<450 \mathrm{~nm})$, ER-1 remained in a ring-closed, non-emissive form. Upon irradiation, the diazoindanone would undergo Wolff rearrangement to give a brightly fluorescent rhodamine dye. ${ }^{10,11}$ We studied the photophysical properties of ER-1 using absorption and fluorescence spectroscopy (Figure 1,b) in phosphate buffered saline (PBS, $\mathrm{pH}=7.4$ ) and measured an approximate 150-fold increase in fluorescence emission after 15 min of irradiation (405 
$\left.\mathrm{nm}, 2 \mathrm{~mW} \mathrm{~cm}{ }^{-2}, 10 \mathrm{~min}\right)$. The emission signal appeared to reach saturation after $15 \mathrm{~min}$ of irradiation under these same conditions (Figure $1, c)$.

a)

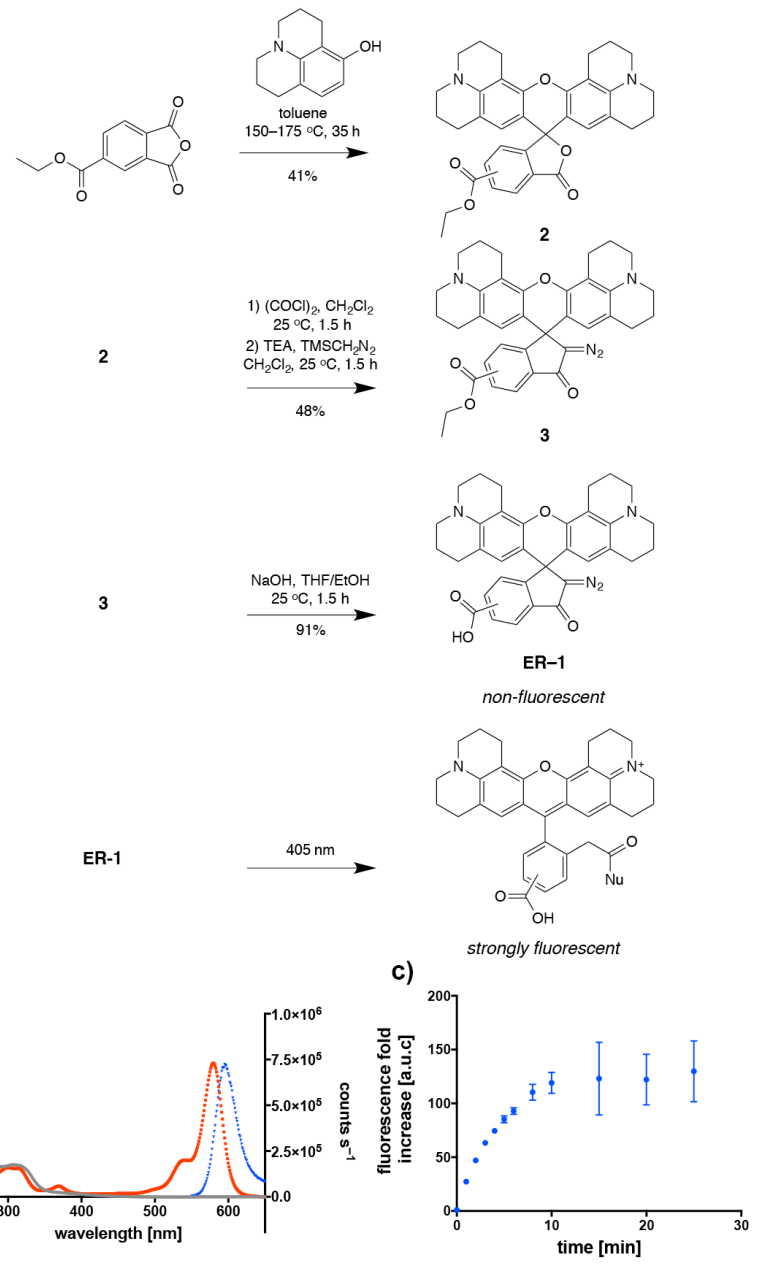

Figure 1. Synthesis and photophysical characterization of ER-1. a) Synthesis and expected photolysis product of ER-1. b) Absorption spectrum (grey solid line) before irradiation and absorption (red squares) and fluorescence (blue triangles) spectra of a solution of ER-1 $(50 \mu \mathrm{M}, \mathrm{PBS}, \mathrm{pH}=7.4)$ after irradiation at $405 \mathrm{~nm}$, $2 \mathrm{~mW} \mathrm{~cm}^{-2}$. c) Fluorescence fold increase of ER-1 (50 $\mu \mathrm{M}, \mathrm{PBS}, \mathrm{pH}$ 7.4) at different irradiation times $\left(405 \mathrm{~nm}, 2 \mathrm{~mW} \mathrm{~cm}{ }^{-2}\right)$.

Because ER-1 provides bright emission signal after photoactivation in bulk, we investigated the photophysical properties of single molecules of ER-1 embedded in a polyvinyl alcohol film (1\% PVA in PBS, ER-1 $\left.=10^{-9} \mathrm{M}\right)$ on a cover glass. PVA films have been used to measure photoswitching of single molecules with good correlation to conventional methods using antibodies attached to glass surfaces. ${ }^{13}$ Films containing ER-1 molecules were hydrated $5 \mathrm{~min}$ prior to imaging with PBS. Single molecules were detected using total internal reflection fluorescence (TIRF) microscopy (Figure 2 and Experimental Section). We applied an initial photoactivation pulse $\left(405 \mathrm{~nm}, 20 \mathrm{~ms}, 2.6 \mathrm{~W} \mathrm{~cm}^{-2}\right)$ followed by continuous wave excitation for reading out the converted molecules $(561 \mathrm{~nm}, 20 \mathrm{~ms}, 0.25$ $\mathrm{kW} \mathrm{cm}^{-2}$ ). A bright, ON state, was evident for molecules irradiated at time $t=0 \mathrm{~s}$ with the photoactivation pulse. After a few seconds, an irreversible dark, OFF state, was induced by permanent photobleaching. An additional experimental set up was attempted, in which sequential photoactivation pulses $\left(405 \mathrm{~nm}, 20 \mathrm{~ms}, 2.6 \mathrm{~W} \mathrm{~cm}^{-2}\right.$ ) were applied every $200 \mathrm{~ms}$ throughout the acquisition. This procedure allowed the detection of multiple particles undergoing sequential photoactivation and photobleaching during the acquisition time, thus facilitating the quantification of a full-ON cycle (Figure $2, a-b$ ).

We determined the half-life $(2 \pm 1.4 \mathrm{~s})$ of the detected group of molecules with a mean of approximately $83,732 \pm 15,415$ photons per molecule (Figure 2, d). These values are comparable with those of commercially available photoactivatable organic dyes. ${ }^{13}$ Because of the high photon count and fast switching times of ER-1, we envision that this molecule would be suitable for live-cell SMLM.
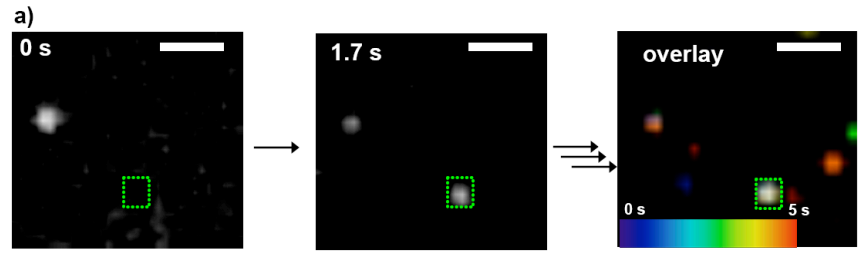

b)
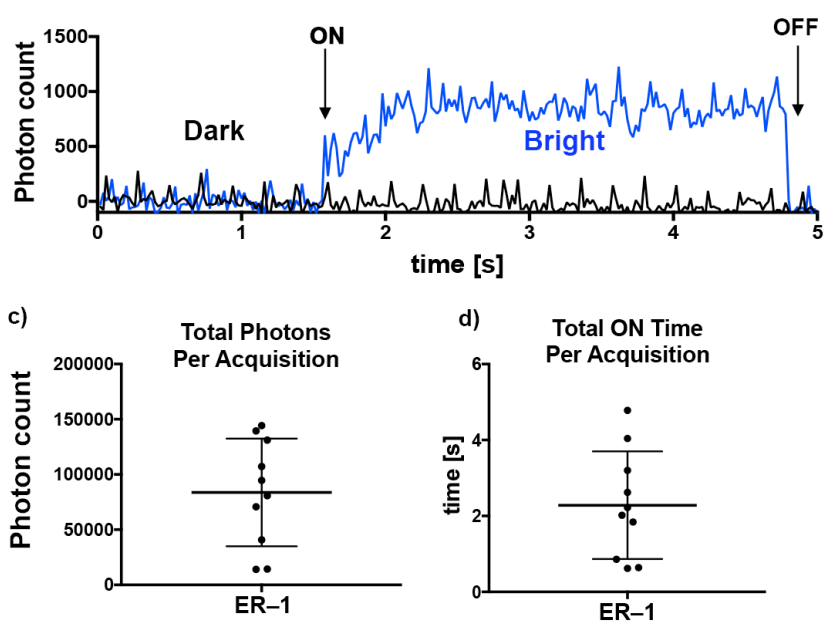

Figure 2. Single-molecule photoactivation in PVA films. a) PVA film containing ER-1 $\left(10^{-9} \mathrm{M}\right)$ in PBS displaying an area containing five molecules at times $0,1.7 \mathrm{~s}$ and an overlay of all time acquisition color-coded in time (0-5 s). The green region of interest depicts a molecule undergoing photoactivation. b) Plot of the total photon count of the selected molecule in panel (a) in time (5 s) within an acquisition following photoirradiation ( $405 \mathrm{~nm}, 20 \mathrm{~ms}, 2.6 \mathrm{~W} \mathrm{~cm}^{-2}$, every $200 \mathrm{~ms}$ ) under continuous wave $561 \mathrm{~nm}$ laser $\left(20 \mathrm{~ms}, 2.6 \mathrm{~W} \mathrm{~cm}^{-2}\right)$ irradiation. The plot displays a complete ON state followed by bleaching after $4.5 \mathrm{~s}$. c) Total photons and d) total ON time per molecule obtained for $N=10$ molecules of ER-1. Mean and standard deviation are displayed. Scale bars: $\mathrm{a}=500 \mathrm{~nm}$.

We next determined whether probe ER-1 targeted any specific subcellular compartment. Live human cervical cancer (HeLa) cells were imaged using a spinning disk microscope to test for membrane permeability, photoactivation efficiency and co-localization with organelle markers. The cells were incubated with ER-1 (10 $\mu \mathrm{M})$ and imaged $\left(\lambda_{\mathrm{ex}}=561 \mathrm{~nm}\right.$ ) before and after irradiation (Figure 3,a-b). A significant increase in fluorescence emission (100-fold) was observed within an intracellular compartment, excluding the cell nucleus, proving permeability across the plasma membrane. To assess the location where 
fluorescent protein, expressed in the ER (Addgene \#36204), mitochondria (Addgene \#36208), Golgi (Addgene \#36205), or lysosomes (Addgene \#110948) and compared to the signal location of ER-1 at a different wavelength. The Pearson's correlation coefficient was determined by comparing the respective cyan images (mTurquoise2) with images from the red imaging channel (ER-1, Figure 3,c-e). The highest correlation was observed with the ER marker $(P=0.8)$, whereas co-localization with mitochondria, Golgi and lysosomes was significantly lower $(P<0.4)$. A closer inspection of the co-localized images revealed that a few mitochondria, particularly around the nucleus were also labeled by ER-1. In the periphery of the cell, however, ER-1 labeled exclusively the thin tubular structures of the ER (Figure 3,d-j). Subsequent analysis of ER morphologies employing ER-1 focused on this region of the cell.
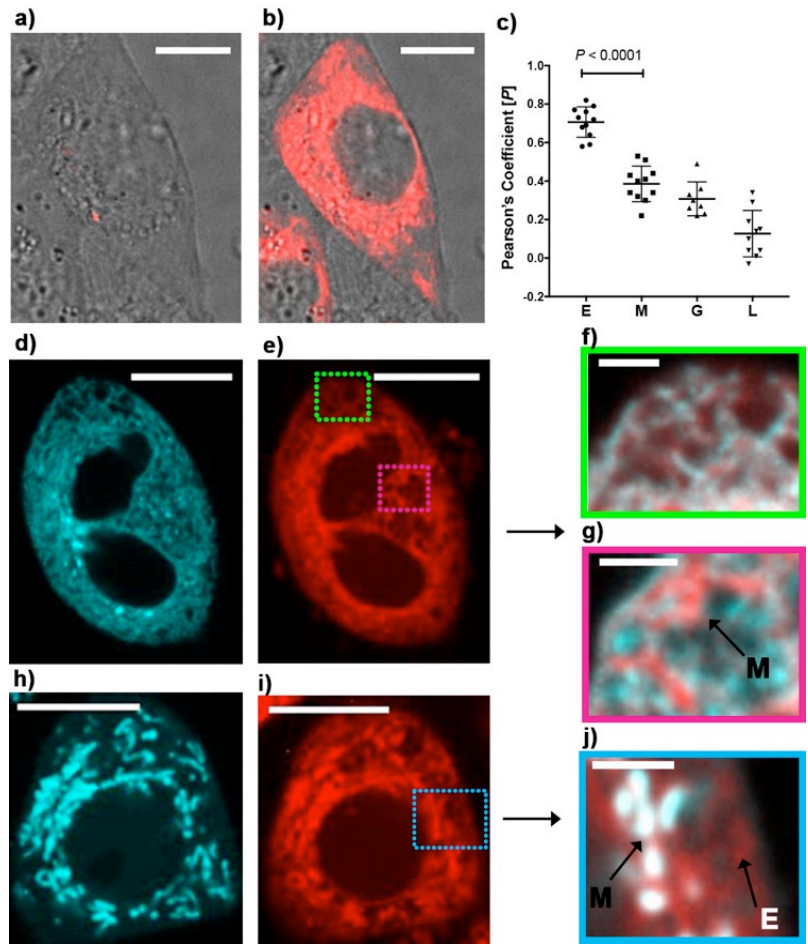

Figure 3. Confocal microscopy and co-localization studies of ER-1. a) Bright-field image of a HeLa cell incubated with ER-1 (10 $\mu \mathrm{M}, 1 \mathrm{~h})$, merged with the red channel $\left(\lambda_{\mathrm{ex}}=561 \mathrm{~nm}\right)$ image, before photoactivation. b) Merged bright-field and fluorescence image $\left(\lambda_{\text {ex }}=561 \mathrm{~nm}\right)$ of the same cell as in panel (a) after photoactivation (405 nm). c) Pearson's correlation coefficients of ER-1 $\left(\lambda_{\mathrm{ex}}=561 \mathrm{~nm}\right)$ with mTurquoise2 $\left(\lambda_{\mathrm{ex}}=445 \mathrm{~nm}\right.$ ) expressed in the ER $(\mathrm{E})$, mitochondria $(\mathrm{M})$, Golgi apparatus (G), or lysosomes (L). A total of $N=10$ cells were selected per condition. Statistical significance was assessed by unpaired, double-tailed Mann-Whitney test. d) Cell expressing fluorescent mTurquoise2-ER marker ( $\lambda_{\mathrm{ex}}=445 \mathrm{~nm}$ ). e) Same cell as in panel (d) displaying emission from ER-1 $\left(\lambda_{\mathrm{ex}}=561 \mathrm{~nm}\right.$ ) after photoactivation (405 $n m$ ). The green square represents an area in the periphery. $f$ ) Overlay of the green region of interest in panels (d) and (e) revealing specific ER co-localization in the periphery of the cell. g) Overlay of the magenta square depicting labeling of mitochondria close to the nucleus. h) Cell expressing fluorescent mTurquoise2mitochondria marker ( $\left.\lambda_{\mathrm{ex}}=445 \mathrm{~nm}\right)$. I) Same cell as in panel (h) displaying emission from ER-1 $\left(\lambda_{\mathrm{ex}}=561 \mathrm{~nm}\right)$ after photoactivation $(405 \mathrm{~nm})$. The blue square represents area in the periphery and interior of the cell. J) Overlay of the blue region of interest in panels ( $h$ ) and (i) depicts significant co-localization with mitochondria in the interior of the cell, but poor co-localization in the periphery. Scale bars: a-b, d-e, h-I = $5 \mu \mathrm{m} ; \mathrm{f}-\mathrm{g}, \mathrm{j}=1 \mu \mathrm{m}$.

SMLM experiments were carried out employing HeLa cells treated with ER-1. The concentration of probe ER-1 was decreased to $1 \mu \mathrm{M}$ to avoid overlapping signals of single emitters within a diffraction-limited spot, which is essential to obtain images free of artifacts. Under these imaging conditions, we could detect single molecules of ER-1 in live cells with a localization precision of $42 \pm 12 \mathrm{~nm}$ (Figure S1, Supporting Information) in the lateral axis over 10,000 frames (250 s, Figure S1). ER tubules and sheets were evident even when a single super-resolved image was obtained using all 10,000 frames (Figure 4,a), despite the potential motion blur. By enhancing the time resolution to $25 \mathrm{~s}$ (1,000 frames per superresolved image), we could observe the fine structures and porous sheets that resemble previously reported ER morphologies (Figure $5, b){ }^{3}$ This $2 \mathrm{D}$ time-lapse demonstrates the highly dynamic structure of the ER and suggests fast movements, breakage (75 s) and loop formation (125-175 s) of the tubular structures of the ER (white arrows, Figure 4,b). Importantly, we did not detect any morphological changes or evidence of membrane disruption after these SMLM experiments (10,000 frames), indicating that the cells remained viable during these short acquisitions.
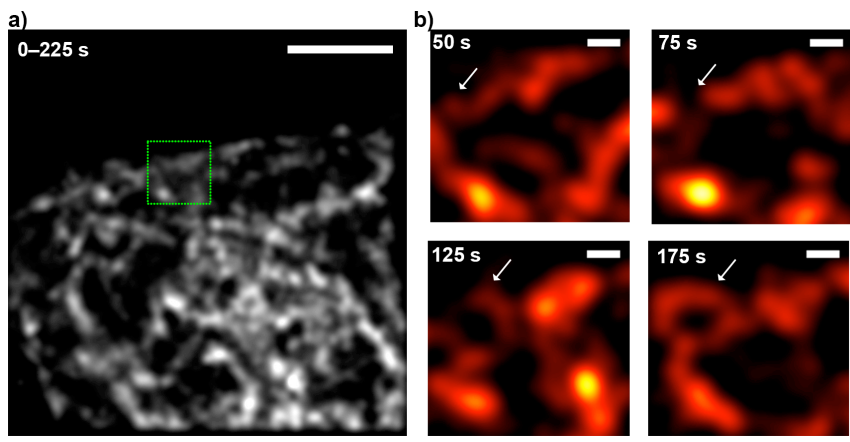

Figure 4. 2D Super-resolution imaging of a HeLa cell with probe ER-1 (1 $\mu \mathrm{M})$. a) Overlay of all 10,000 frames obtained in a $225 \mathrm{~s}$ acquisition. Photoactivation was achieved with $405 \mathrm{~nm}$ ( $20 \mathrm{~ms}, 2.6 \mathrm{~W} \mathrm{~cm}^{-2}$ pulse every $200 \mathrm{~ms}$ ) readout emission was obtained using $561 \mathrm{~nm}$ light $\left(2.6 \mathrm{~W} \mathrm{~cm}^{-2}\right)$. The green dotted box represents a region of interest selected in the periphery of the cell. b) Time-lapse sequence of the region of interest in panel (a) shown with a red look up table (LUT) depicting the breakage of a tubule followed by the formation of a loop in a later time frame (white arrows) representing the ER at nanoscale. Scale bars: $a=5 \mu \mathrm{m} ; b=500 \mathrm{~nm}$.

Because of the dynamic movement of the ER in three dimensions, we hypothesized that a detailed analysis of the nanometric structure could only be obtained by $3 \mathrm{D}$ imaging with a high time resolution. We performed 3D SMLM employing adaptive optics (3D SMLM, Experimental Section) and obtained similar localization precision as before $(49 \pm 10 \mathrm{~nm}$, Figure 5a), which represents nearly an order magnitude improvement over diffraction-limited optical microscopy (Figure 5,b-c). A super-resolved, 3D image was reconstructed employing 2,000 acquisition frames (time resolution $=20 \mathrm{~s}$, Figure 5,c). This $3 \mathrm{D}$ image reveals the presence of multiple holes $\left(\mathrm{H}_{1-2}\right)$ throughout the ER network. However, when dissecting the axial plane in steps of $200 \mathrm{~nm}$, we observed formation of larger holes (merged $\mathrm{H}_{1-2}$, at $\mathrm{z}=-200 \mathrm{~nm}$ ) and closure of the pore (at $\mathrm{z}=$ $+200 \mathrm{~nm}$, Figure 5,d). These nanometric details of the dynamic topology 
of the ER visible at this temporal and spatial resolution highlight the importance of 3D imaging enabled by probe ER-1. A 3D reconstruction reveals the existence of nanometric holes ( $<250 \mathrm{~nm}$ diameter) and shows the porous, concave structure that the ER structure can adopt (Figure 5,e and Supplementary Movie 1).

a)
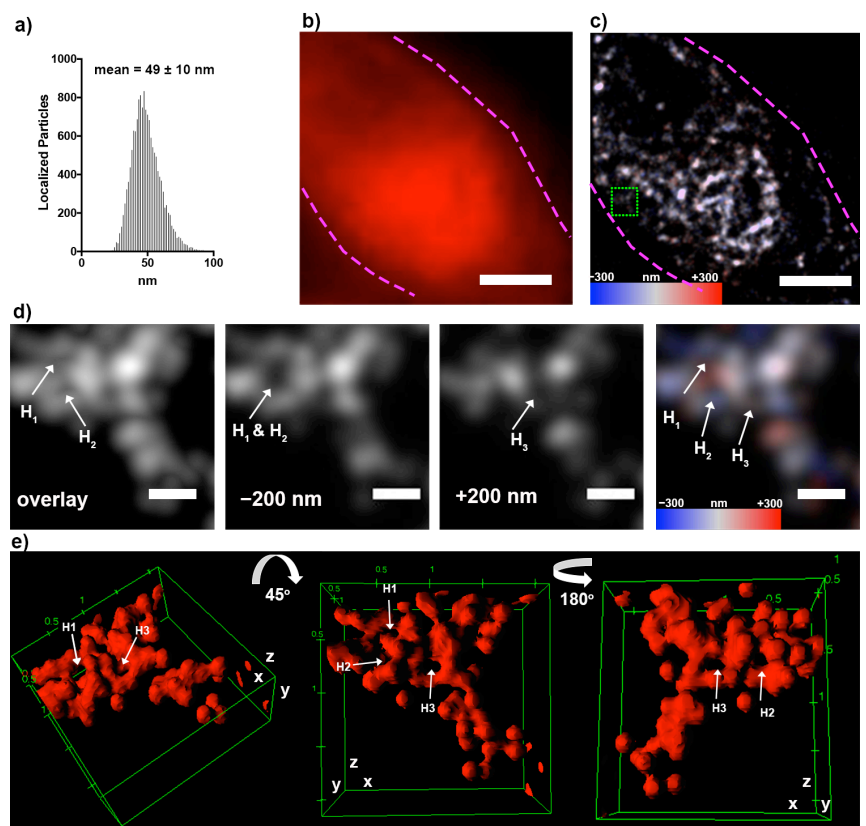

Figure 5. 3D SMLM of ER-1 in live HeLa cells. a) Localization precision of single molecules. b) Diffraction-limited, deconvolved image obtained from merging all signals found in 2000 frames (20 s) of acquisition. c) Super-resolved counterpart of HeLa cell in panel (b). All localizations were color-coded with an axial depth of $600 \mathrm{~nm}$. A green region of interest was selected in the periphery of the cell were fine ER structures were evident. d) Region of interest in panel (c) depicting presence of nanometric holes ( $\mathrm{H}_{1-2}$, white arrows) at different axial depths. A depth color-coded image displays the respective distance to which extensions are located within the field of view. e) 3D reconstruction of the same field of view as in (d) depicting the porous morphology of the ER and the presence of real holes $\left(\mathrm{H}_{1-3}\right)$. Scale bars: $b-c=5$ um; d-e: $500 \mathrm{~nm}$.

\section{Conclusions}

We developed a simple diazoindanone rhodamine probe, which is useful for imaging ER nanostructures structures with 2D and 3D SMLM. We characterized the photophysics of this probe both in bulk and at the single-molecule level. Employing this probe, we were able to observe fine structures of the ER and their nanometric pores $(<250 \mathrm{~nm})$ with 2D SMLM and were able to discern between concave cavities from real holes using 3D SMLM reconstructions. This simple probe enables the study of the structure of cellular organelles and can help elucidate nanometric morphological changes in live, non-genetically modified cells.

\section{Experimental Section}

\section{Materials and Methods}

Reagents (ABCR, Acros, Aldrich, Fluka, Lancaster, and TCl) were purchased as reagent grade and used without further purification. Solvents for flash column chromatography (FC), and for reactions were of HPLC and technical grade respectively. Dry solvents for reactions were purified by a solvent drying system (LC Technology Solutions Inc. SP-105) under nitrogen atmosphere $\left(\mathrm{H}_{2} \mathrm{O}\right.$ content $<10$ ppm as determined by KarlFischer titration). All other solvents were purchased in p.a. quality. For all aqueous solutions, ultra-pure deionized water was used. Reactions under exclusion of air or water were performed in oven-dried glassware and in a $\mathrm{N}_{2}$ atmosphere. Flash column chromatography (FC) was carried out using silica gel (particle size: 40-63 $\mu \mathrm{m}, 230-400$ mesh ASTM; Silicycle) or using a Büchi Reveleris ${ }^{\circledast}$ PREP purification system with HPLC grade solvents. Analytical thin layer chromatography (TLC) was performed on aluminum sheets or glass plates coated with silica gel $60 \mathrm{~F}_{254}$ (Merck); visualization with a UV lamp $(254 \mathrm{~nm})$. Evaporation under reduced pressure was performed at $45^{\circ} \mathrm{C}$ and $900-10$ mbar. ${ }^{1} \mathrm{H}$ and ${ }^{13} \mathrm{C}$ nuclear magnetic resonance (NMR) spectra were recorded on Bruker AV 400 and Bruker AV-NEO 500 recorded at 400 or $500 \mathrm{MHz}\left({ }^{1} \mathrm{H}\right)$ and 100 or $125 \mathrm{MHz}$ $\left({ }^{13} \mathrm{C}\right)$, respectively. Chemical shifts $(\delta)$ are reported in ppm downfield from tetramethylsilane using the residual solvent signals as an internal reference. For ${ }^{1} \mathrm{H}$ NMR, coupling constants $J$ are given in $\mathrm{Hz}$ and the resonance multiplicity is described as $s$ (singlet), $d$ (doublet), $t$ (triplet), $q$ (quartet), $\mathrm{m}$ (multiplet). All spectra were recorded at $25{ }^{\circ} \mathrm{C}$. Mass spectrometry (MS) and high-resolution mass spectrometry (HR-MS) were performed by the MS-service of the Laboratory for Organic Chemistry at the ETH Zürich on a Waters Micromass AutoSpec-Ultima spectrometer (EI), on a Bruker maXis spectrometer (ESI). Masses are reported in $\mathrm{m} / \mathrm{z}$ units for the molecular ion $\mathrm{M}^{+}$, or $\left[M+\mathrm{H}^{+},[M+\mathrm{Na}]^{+}\right.$or $[M+\mathrm{K}]^{+}$with the corresponding intensities in \%.

\section{Absorption and Fluorescence Spectoscopy}

Spectra were recorded on a Varian Cary-500 Scan spectrophotometer. The spectra were measured in a quartz cuvette (ThorLabs, CV10Q3500) at $25{ }^{\circ} \mathrm{C}$. Quartz cuvettes were irradiated with an LED transilluminator (Home-built) emitting at $405 \mathrm{~nm}$ and an incident intensity of $\sim 2 \mathrm{~mW} \mathrm{~cm}^{-2}$, measured with a power-meter (ThorLabs, PM100D) equipped with a Si photodiode detector (ThorLabs, S120VC). Fluorescence spectroscopy was measured in a Fluorolog 3 fluorimeter (Horiba Jobin-Yvon) fluorimeter with a cuvette sample charger in quartz cuvettes (ThorLabs, CV10Q3500F-E).

\section{Cell Culture and Plating}

HeLa cells (ATCC CCL2) were grown in Dulbecco's Modified Eagle Medium (DMEM) supplemented with fetal bovine serum (FBS, 10\%) and penicillin-streptomycin $(0.1 \%)$, referred to as growth medium at $37^{\circ} \mathrm{C}$ in a $95 \%$ humidity atmosphere under $5 \% \mathrm{CO}_{2}$ environment. Cells were grown to $90 \%$ confluence and seeded onto 8- well Nunc ${ }^{\mathrm{TM}}$ Lab-Tek $^{\mathrm{TM}}$ ॥ chambered cover glass plates (50 000 cells/well) for confocal microscopy, or onto lbdi $\mu$-slide 8 well plates (50 000 cells/well) a day prior to imaging experiments. 


\section{Confocal Microscopy}

Confocal microscopy was done using a Nikon Eclipse Ti light microscope equipped with a Yokogawa spinning-disk confocal scanner unit CSU-W1T2, two sCMOS cameras (Orca Flash 4.0 V2), and a LUDLPrecision2 stage with a piezo focus. Diode-pumped solid-state lasers (DPSS) were used as light sources: $405 \mathrm{~nm}(120 \mathrm{~mW}$, 50\% laser power, $1 \mathrm{~s})$ and $561 \mathrm{~nm}(200$ $\mathrm{mW}, 70 \%, 0.5 \mathrm{~s})$. The exposure time, acquisition time, and laser powers were kept constant unless stated otherwise. All images were collected using a $100 \times$ CFI Apo TIRF (NA = 1.49) objective with oil-immersion. Emitted light was filtered using the filters: BP 450/50, BP 470/24, and BP $605 / 52$. The microscope was operated using VisiVIEW (Metamorph) software. Quantification of fluorescence intensity was carried out using Fiji. ${ }^{14}$ The cell body was selected as the region of interest (ROI), and the integrated intensity within the ROI was measured. An additional ROI of the same size and shape was used to obtain the integrated intensity of the background (region with no cell). The background intensity was subtracted from that of the cell-containing ROI. All data sets were analyzed by unpaired, two-tailed, Student's $t$ test. The statistical analyses were carried out using Prism 6 (GraphPad). Error bars represent standard deviation, $\mathrm{P}$-values are given, and results are depicted as mean.

\section{Colocalization Studies}

Thresholding of the images was done selecting the active sequence input, followed by a manual threshold treated as percentages and calibrated with an output as a single ROI. Pearson's correlation coefficients were obtained using the thresholding studio (ICY).

\section{Super Resolution Microscopy and SMLM}

Experiments were done with HeLa cells plated in phenol red-free growth medium (Gibco). Cells were incubated with compound ER-1 for $1 \mathrm{~h}$ washed with PBS $(0.25 \mathrm{~mL})$ before imaging. Cells were imaged using a Nikon N-STORM, microscope (Nikon, UK Ltd.) equipped with an SR Apochromat TIRF 100x 1.49 N.A. oil immersion objective lens. Excitation laser at a wavelength $\lambda=561 \mathrm{~nm}$ illuminated the sample in HILO mode. ${ }^{15}$ Fluorescence was detected with an iXon DU897 (Andor) EM-CCD camera $\left(16 \times 16 \mu \mathrm{m}^{2}\right.$ pixel size) for 2D SMLM and a Hamamatsu Orca Flash 4 v3 $\left(6.5 \times 6.5 \mu \mathrm{m}^{2}\right.$ pixel size) for 3D SMLM with an exposure time of $20 \mathrm{~ms}$. An in-built focus-lock system (perfect focus system, PFS) was used to prevent axial drift of the sample during data acquisition. The emission was collected and passed through a laser QUAD filter set for TIRF applications (Nikon C-NSTORM QUAD 405/445/561) comprising laser clean-up, dichroic and emission filters. The laser excitation at $561 \mathrm{~nm}$ had a power density of $0.25 \mathrm{~kW} \mathrm{~cm}^{-2}$. 2D and 3D SMLM camera frames were recorded at 46 or 100 frames per second, respectively and for the later an adaptive optics plug and play accessory MicAO 3DSR for SMLM (Imagine Optic, France) was inserted between the microscope side port and the SCMOS, Hamamatsu Orca Flash 4 v3 camera. As a second step, a controllable and aberration-free astigmatism was introduced to the point spread function (PSF) of single molecules, which allowed us to perform 3D SMLM based on astigmatism by determining the $\mathrm{z}$ positions of the fluorescent molecules based on the ellipticity of their PSF. ${ }^{16} 3 \mathrm{D}$ calibration was performed with fluorescent $0.2 \mu \mathrm{m}$ beads (Tetraspect ${ }^{\mathrm{TM}}$, Invitrogen). From each image stack, a reconstructed super-resolved image was generated using Thunderstorm $(\mathrm{FIJI})^{17}$ and $3 \mathrm{D}$ projections were made using 3D Viewer (FIJ).

\section{Supplementary Material}

Supporting information for this article is available on the WWW under http://dx.doi.org/10.1002/MS-number.

\section{Acknowledgements}

This work was supported by ETH Zurich (grant ETH-02 16-1). We acknowledge the Scientific Center for Optical and Electron Microscopy (ScopeM, ETH Zurich) for access to microscopy facilities.

\section{Author Contribution Statement}

E. H. R and S. P performed all experiments and analyzed data. P. R.-F. designed and supervised research and analyzed data. E. H. R. and P. R.-F. wrote the manuscript with input from S.P.

\section{References}

M. Hofmann, C. Eggeling, S. Jakobs, S. W. Hell, 'Breaking the diffraction barrier in fluorescence microscopy at low light intensities by using reversibly photoswitchable proteins', Proc. Natl. Acad. Sci. U. S. A. 2005, $102,17565-17569$.

2. X. Huang et al., 'Fast, long-term, super-resolution imaging with Hessian structured illumination microscopy', Nat. Biotechnol. 2018, 36, 451-459.

3. J. Nixon-Abell et al., 'Increased spatiotemporal resolution reveals highly dynamic dense tubular matrices in the peripheral ER', Science. 2016, 354, aaf3928.

4. L. D. Lavis, R. T. Raines, 'Bright ideas for chemical biology', ACS Chem. Biol. 2008, 3, 142-155

5. J. B. Grimm et al., 'Bright photoactivatable fluorophores for singlemolecule imaging', Nat. Methods 2016, 13, 985-988.

D. T. Burnette, P. Sengupta, Y. Dai, J. Lippincott-Schwartz, B. Kachar, 'Bleaching/blinking assisted localization microscopy for superresolution imaging using standard fluorescent molecules', Proc. Natl. Acad. Sci. U. S. A. 2011, 108, 21081-21086.

7. S. Uno et al., 'A spontaneously blinking fluorophore based on intramolecular spirocyclization for live-cell super-resolution imaging', Nat. Chem. 2014, 6, 681-689.

8. H. Takakura et al., 'Long time-lapse nanoscopy with spontaneously blinking membrane probes', Nat. Biotechnol. 2017, 35, 773-780.

9. H. Deschout et al., 'Precisely and accurately localizing single emitters in fluorescence microscopy', Nat. Methods 2014, 11, 253-266.

10. V. N. Belov, C. A. Wurm, V. P. Boyarskiy, S. Jakobs, S. W. Hell, 'Rhodamines NN: A novel class of caged fluorescent dyes', Angew. Chem. Int. Ed. 2010, $49,3520-3523$

11. E. A. Halabi, Z. Thiel, N. Trapp, D. Pinotsi, P. Rivera-Fuentes, 'A photoactivatable probe for super-resolution imaging of enzymatic activity in live cells', J. Am. Chem. Soc. 2017, 139, 13200-13207. 
F. Huang et al., 'Ultra-high resolution 3D imaging of whole cells', Cell 2016, 166, 1028-1040.

A. M. Bittel et al., 'Methodology for quantitative characterization of fluorophore photoswitching to predict superresolution microscopy image quality.', Sci. Rep. 2016, 6, 29687.

14. J. Schindelin et al., 'Fiji: an open-source platform for biological-image analysis', Nat. Methods 2012, 9, 676-682 .

15. M. Tokunaga, N. Imamoto, K. Sakata-Sogawa, 'Highly inclined thin illumination enables clear single-molecule imaging in cells', Nat. Methods 2008, 5, 159-161 .

16. B. Huang, W. Wang, M. Bates, X. Zhuang, 'Three-dimensional superresolution imaging by stochastic optical reconstruction microscopy', Science. 2009, 319, 5864 .

17. M. Ovesný, P. Kř́žžek, J. Borkovec, Z. Švindrych, G. M. Hagen, 'ThunderSTORM: A comprehensive ImageJ plug-in for PALM and STORM data analysis and super-resolution imaging', Bioinformatics 2014, 30, 2389-2390. 